\title{
ESTUDIO ETNOBOTÁNICO Y FITOQUÍMICO DE HOJAS DE Brassica oleracea L. "COL MORADA"
}

\author{
PHYTOCHEMICAL AND ETHNOBOTANICAL STUDY LEAVES Brassica \\ oleracea L. "PURPLE CABBAGE"
}

\author{
'Gustavo Adolfo Fernández Rebaza, ${ }^{1}$ Yoselin Milagros Chambi Velasquez, ${ }^{1}$ José Miguel Ayme Huamani, \\ 'María Eugenia Quiñones Huayaney, 'Jorge Luis Tolentino Chavez, ${ }^{2}$ Víctor Elmo Miranda García, \\ ${ }^{1}$ Pablo Enrique Bonilla Rivera
}

\begin{abstract}
RESUMEN
Se realizó un estudio etnobotánico y fitoquímico de Brassica oleracea L. "col morada". Teniendo como objetivos detectar metabolitos secundarios fenólicos. La especie fue ubicada y recolectada en la Región Junin. En el análisis fitoquímico del extracto etanólico se detectó flavonoides, antocianósidos y otros. Se realizó cromatografía en capa fina en los diferentes sistemas de solventes (cloroformo:metanol); luego, un fraccionamiento con diclorometano, etanol y agua mediante cromatografía en columna rápida, obteniéndose en el subextracto etanólico presencia de flavonoides y otros compuestos fenólicos a la luz UV, que fue corroborado al utilizar reveladores como amoniaco y tricloruro férrico. En la determinación estructural mediante espectroscopía UV-visible del subextracto etanólico se obtuvo tres probables flavonoides: dos de núcleo flavona y uno de flavanona.
\end{abstract}

Palabras clave: Brassica oleracea L., flavonoides, flavonas, cromatografia, espectroscopía UV-visible.

\section{ABSTRACT}

Ethnobotanical study and phytochemical of Brassica oleracea L. "purple cabbage" was performed. The objectives were detected phenolic secondary. The species was located and collected in Junin region. Phytochemical analysis of the ethanol extract detected flavonoids, anthocyanins and other. Thin Layer Chromatography was performed in different solvents (chloroform: methanol); then, a fractionation with dichloromethane, ethanol and water by flash chromatography to get flavonoids and other phenolic compounds in the ethanolic extract sub with UV light, which was confirmed by using developers as ammonia and ferric trichloride. The structural determination by UV-visible spectroscopy of ethanolic extract sub obtained three probable flavonoids: two flavone core and one flavanone.

Keywords: Brassica oleracea L., flavonoids, flavones, chromatography, UV-visible spectroscopy.

\section{INTRODUCCIÓN}

Actualmente el estudio de flavonoides ha cobrado alta importancia porque la mayoría de ellos protegen el daño producido por agentes oxidantes, por ello la búsqueda de plantas ' con estos compuestos tales como Brassica oleracea $\mathrm{L}$. la cual es una planta originaria de Europa (Inglaterra, Dinamarca, Francia, España, Holanda). ${ }^{2}$ Usada comúnmente para el tratamiento de inflamaciones crónicas de la membrana del estómago y úlcera gástrica, además contiene gran número de compuestos anticancerígenos y antioxidantes. Existen variedades en el Perú como la "col blanca", "col verde", "col morada o roja". 2 En el presente estudio se utilizó la planta Brassica oleracea L. "col morada" para una evaluación etnobotánica referencial y una fitoquímica según Olga Lock, ${ }^{4}$ con algunas variantes para el extracto etanólico de hojas. Luego se utilizaron técnicas cromatográficas que permitieron separar compuestos del extracto utilizando silicagel $60 \mathrm{G}$, el mejor adsorbente para separación de sustancias de naturaleza polar. Se proponen estructuras químicas, utilizando espectroscopía UV-visible, los que posiblemente sean responsables de la actividad citotóxica sobre células cancerígenas.

\section{MATERIALES Y MÉTODOS}

Estudio etnobotánico e identificación morfológica de la planta

La "col morada" se recolectó en Junín-Perú a una altitud de $3050 \mathrm{msnm}$ en el mes de agosto, con una humedad relativa de $70 \%$ y temperatura ambiental de $17^{\circ} \mathrm{C}$. Fue clasificada en el Museo de Historia Natural de la UNMSM. Las hojas son alternadas, simples y sin estípulas, con frecuencia lobuladas de color verde (cubren la cabeza). Se tomó las

'Instituto de Ciencias Farmacéuticas y Recursos Naturales "Juan de Dios Guevara", Facultad de Farmacia y Bioquímica de la Universidad Nacional Mayor de San Marcos.

${ }^{2}$ Colaborador 
Fernández, G. et al. Estudio etnobotánico y fitoquimico de hojas de Brassica oleracea L. "col morada".

hojas que se encontraban en la cabeza (parte comestible) que en su totalidad tenían color morado y de aspecto liso, pesando aproximadamente $1 \mathrm{~kg}$.

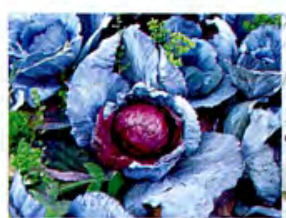

A

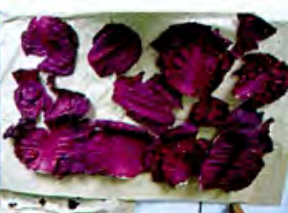

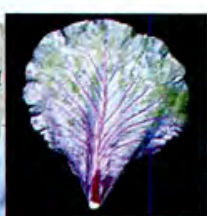

C
Figura 1. A) Col morada o Repollo presenta hojas verdes rodeando la cabeza que es de color morado, B) Hojas de la cabeza del repollo, C) Morfología de hojas externas de la planta

Obtención de la muestra:

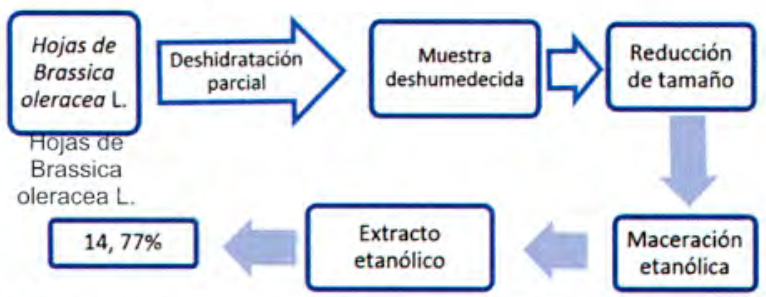

Figura 2. Método operatorio para obtención del extracto seco.

\section{Tamizaje fitoquímico}

Se realizó en el Laboratorio de Farmacognosia y Medicina Tradicional en la Facultad de Farmacia y Bioquímica de la UNMSM, de acuerdo al método descrito por Olga Lock ${ }^{4}$ por ofrecer mayor reproducibilidad y ser de fácil ejecución.

Según este método se realizó la marcha del extracto etanólico, utilizándose los reactivos de Molish, $\mathrm{FeCl}_{3}$, Gelatina $1 \%$, Shinoda, $\mathrm{H}_{2} \mathrm{SO}_{4 \mathrm{cc}}$, Dragendorff, Mayer, Vainillína clorhídrico entre otros. (Tabla 1).

\section{Técnicas cromatográficas}

Realizadas en el laboratorio del Instituto de Ciencias Farmacéuticas y Recursos Naturales "Juan de Dios Guevara", Facultad de Farmacia y Bioquímica.

\section{Cromatografía en capa fina analítica (CCF)}

Se realizó cromatografía en capa fina analítica del extracto etanólico total, se sembró la muestra de extracto semisólido disuelto en etanol en varios sistemas de solventes de cloroformo: metanol en las proporciones 3:2, 1:1, 1:2, 2:3 y 1:3. Se reveló las cromatoplacas a la lámpara de luz UV 365 nm y $254 \mathrm{~nm}, \mathrm{FeCl}_{3}$ y vapores de amoníaco.

\section{Cromatografia en columna rápida}

Se fraccionó $50 \mathrm{~g}$ del extracto etanólico total mediante cromatografía en columna rápida utilizando Silicagel para cromatografía en columna, con los siguientes eluyentes: diciorometano, etanol y agua, obteniéndose los subextractos correspondientes.

Se realizó CCF analítica del subextracto etanólico y acuoso en cromatoplacas de silicagel 60-G utilizando como sistema de solventes cloroformo: metanol (3:1), luego se reveló en lámpara UV $365 \mathrm{~nm}$ y $254 \mathrm{~nm}$, vapores de amoníaco y tricloruro férrico.

\section{Cromatografia en capa fina a escala preparativa}

Se realizó la cromatografía en capa fina a escala preparativa de las muestras para aislar los componentes en el sistema de solventes cloroformo: metanol 3:1 del subextracto etanólico y acuoso.

\section{Determinación de estructuras químicas}

Luego de realizar la CCF a escala preparativa se procedió a la desorción de las manchas correspondientes. La silicagel con la mancha correspondiente se disolvió en alcohol etilico Q.P. y se centrifugó. Para separar los componentes se usó la centrífuga modelo Rotomix 32 a 2000 RPM, tomándose el sobrenadante y vertiéndolo en viales debidamente rotulados.

Los componentes químicos obtenidos fueron determinados mediante espectroscopía UV, en un rango de 200$400 \mathrm{~nm}$ usando el equipo Thermos cientific modelo Helios Zeta y el programa Vision lite, en el Centro de Producción Farmacéutica (CENPROFARMA) de la Facultad de Farmacia y Bioquímica de la UNMSM.

\section{RESULTADOS}

\section{Tamizaje fitoquímico}

Tabla 1. Resultados del tamizaje fitoquímico de Brassica oleracea L.

\begin{tabular}{|c|c|c|}
\hline Metabolito & Reacción & $\begin{array}{l}\text { Resultado } \\
\text { Extracto total }\end{array}$ \\
\hline \multirow[t]{3}{*}{ Carbohidratos } & Molish & ++ \\
\hline & Benedict & +++ \\
\hline & Fehling & ++ \\
\hline Compuestos Fenólicos & $\mathrm{FeCl}_{3}$ & +++ \\
\hline \multirow[t]{2}{*}{ Taninos } & Gelatina & ++ \\
\hline & Agua de Bromo & + \\
\hline Triterpenoides y Esteroides & Lieberman-Burchard & ++ \\
\hline Flavonoides & Shinoda & +++ \\
\hline $\begin{array}{l}\text { Antocianinas y Flavonoides } \\
\text { Catéquicos }\end{array}$ & Rosenheim & +++ \\
\hline \multirow{2}{*}{$\begin{array}{c}\text { Antocianósidos (Heterósidos } \\
\text { Flavonoides) }\end{array}$} & $\mathrm{H}_{2} \mathrm{SO}_{4 \mathrm{CCC}}$ & +++ \\
\hline & $\mathrm{NaOH} 30 \%$ & ++ \\
\hline Catequinas Glicosiladas & Vainillín clorhídrico & +++ \\
\hline $\begin{array}{l}\text { Naftaquinonas, Antraquinonas y } \\
\text { Antronas }\end{array}$ & Borntrager & + \\
\hline $\begin{array}{c}\text { Aminoácidos Libres y Grupos } \\
\text { Amino }\end{array}$ & Ninhidrina & ++ \\
\hline \multirow[t]{6}{*}{ Alcaloides } & Dragendorff & +++ \\
\hline & Mayer & ++ \\
\hline & Bertrand & +++ \\
\hline & Bouchardat & ++ \\
\hline & Sonnenschein & + \\
\hline & Popoff & ++ \\
\hline Cumarinas & $\mathrm{NH}_{4} \mathrm{OH}_{c \mathrm{c}}$ & + \\
\hline
\end{tabular}

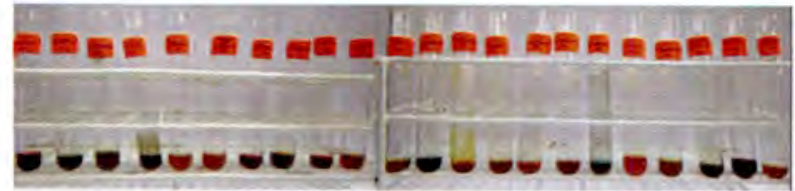

Figura 3. Tamizaje fitoquímico del extracto etanólico total de Brassica oleracea L. 
Fernández, G. et al. Estudio etnobotánico y fitoquímico de hojas de Brassica oleracea L. "col morada".

\section{Cromatografia en capa fina analítica}

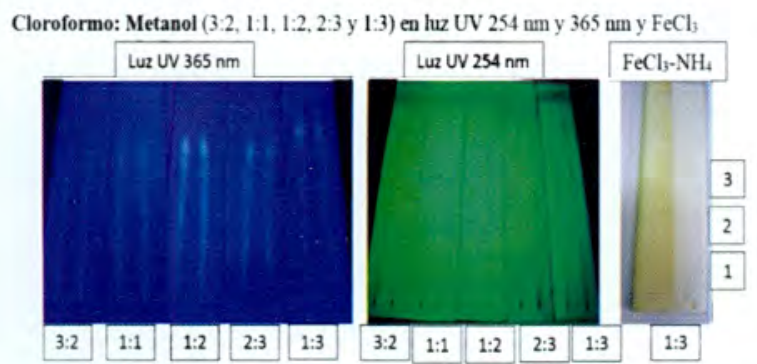

Figura 4. Cromatografía en capa fina analítica del extracto etanolico total de hojas de Brassica oleracea L. Revelado con: lámpara luz UV $365 \mathrm{~nm}, \mathrm{UV} 254 \mathrm{~nm}, \mathrm{FeCl}_{3} \mathrm{y} \mathrm{NH}_{4}$

\section{Cromatografia en capa fina analítica luego de la colum-} na rápida

Total: $50 \mathrm{~g}$ de extracto, Subextracto alcohólico: (15,06\%), Subextracto acuoso: $(37,2 \%)$.

Cloroformo: Metanol (3:1) en lámpara de luz UV $365 \mathrm{~nm}$ y $254 \mathrm{~nm}$ y FeCl 3
Luz UV $365 \mathrm{~nm}$

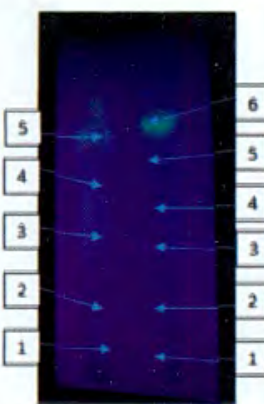

Luz UV $254 \mathrm{~nm}$

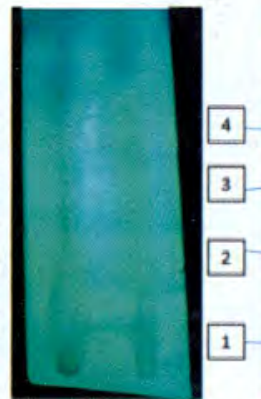

$\mathrm{FeCl}_{1}$

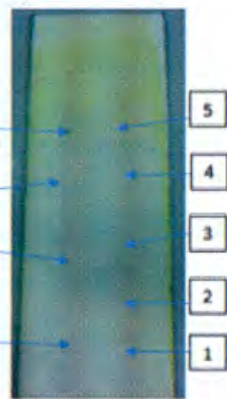

Figura 5. Cromatografía en capa fina analítica comparativa de los sub extractos acuoso y alcohólico de hojas de Brassica oleracea $\mathrm{L}$.

\section{Cromatografia en capa fina a escala preparativa}

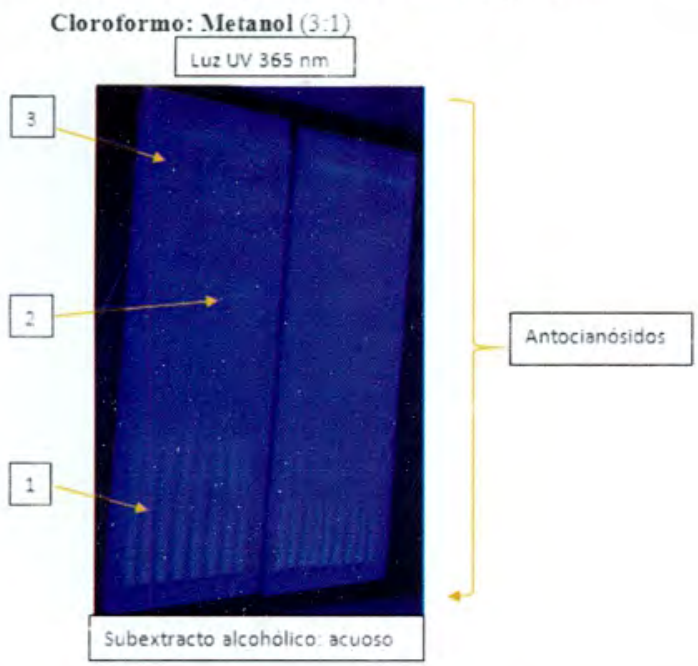

Figura 6. Cromatografía en capa fina a escala preparativa del subextracto alcohólico y acuoso de hojas de Brassica oleracea L.

\section{Propuesta de estructuras químicas}

Del subextracto etanólico se obtuvieron 3 compuestos y se proponen sus estructuras químicas mediante comparación con lo publicado por Mabry et al.

\section{Flavonas:}<smiles>COc1ccc(-c2cc(=O)c3c(O)c(O)c(O)cc3o2)cc1OC</smiles>

Figura 7. $\mathrm{N}^{\circ} 1 \lambda_{\max }^{\mathrm{EOH}}=260275330 \mathrm{~nm}$ 5,6,7-trihidroxi-3', $4^{\prime}$-dimetoxiflavona<smiles>COc1ccc(-c2cc(=O)c3c(O)cc(O)c(O)c3o2)cc1</smiles>

Figura 8. $\mathrm{N}^{\circ} 2 \lambda_{\max }^{\mathrm{ErOH}}=260280320 \mathrm{~nm}$ $5,7,8$-trihidroxi-4'-metoxiflavona

Flavanonas:<smiles>COc1c(O)cc(C2CC(=O)c3c(O)cc(O)cc3O2)cc1O</smiles>

Figura 9. $\mathrm{N}^{\circ} 3 \quad \lambda_{\max }^{\mathrm{EFH}}=260290330 \mathrm{~nm}$ 3',5',5,7-tetrahidroxi-4'-metoxiflavanona

En el extracto acuoso se detectó presencia de anto cianinas, mediante la marcha fitoquímica y fluorescencia característica en cromatografía en capa fina.

\section{DISCUSIÓN}

El screening fitoquímico del extracto etanólico de Brassica oleracea L. detecta la presencia de compuestos fenólicos, flavonoides, glicósidos, taninos, antocianinas y alcaloides. ${ }^{4}$ 
Por ensayos cromatográficos se identifican compuestos fenólicos y flavonoides, utilizando como adsorbente silicagel $60-\mathrm{G}$ y como fase móvil $\mathrm{CHCl}_{3}$ : $\mathrm{MetOH}(3: 1)$; observando los cromatogramas a la lámpara de luz UV 254 $\mathrm{nm}$ y $365 \mathrm{~nm}$ se visualiza las manchas correspondientes a flavonoides, además de reveladores cromogénicos como amoniaco y $\mathrm{FeCl}_{3}$ al $5 \%$ que confirman dicha presencia de compuestos fenólicos. ${ }^{4.5}$

Los flavonoides son aislados y purificados mediante cromatografía en capa fina a escala preparativa, utilizándose como adsorbente silicagel 60-G, como sistema de solventes cloroformo: metanol ( $3: 1$ y $1: 3)$, y se observa los cromatogramas a la lámpara de luz UV a $254 \mathrm{~nm}$ y $365 \mathrm{~nm}^{4,5}$

Se separan tres fracciones a partir del subextracto etanólico los cuales por métodos espectroscópicos UVvisible, y por comparación con espectros publicados en Mabry, permiten proponer estructuras químicas como flavonoides de núcleo flavona y flavanona. Para acercarnos más a la estructura real se tendría que evaluar con reactivos de desplazamiento ${ }^{7}$ y de esta manera confirmar dichas estructuras químicas.

Además, los compuestos fenólicos son unos de los principales metabolitos secundarios de las plantas, por tanto, son productos biosintetizados en las plantas, en las que desarrollan diversas funciones, como la protección frente a condiciones externas. Se encuentran en casi todos los alimentos de origen vegetal aportando color, sabor amargo, astringencia y/o aroma, siendo su distribución muy diversa dentro de las plantas (hojas, flores, frutos, semillas, tallos, raíces). Los compuestos fenólicos, especialmente los flavonoides, poseen diferente actividad biológica, pero las más importantes son la actividad antioxidante y el efecto inhibidor de varias clases de tumores.

Finalmente de acuerdo a otros trabajos el contenido de polifenoles y otros componentes fitoquimicos en coles puede ser influenciado por varios factores tales como cultivos, condiciones climáticas, madurez en condiciones de cosecha y almacenamiento. Así en el caso de compuestos fenólicos, que son especies altamente reactivas, el método de preparación de la muestra es muy importante para su deteccion. ${ }^{9,10}$

\section{CONCLUSIONES}

1. Se identificó la muestra en estudio como Brassica oleracea L. "col morada".

2. Se detectaron flavonoides en el subextracto etanólico de hojas de Brassica oleracea L. "col morada" mediante ensayos cromatográficos y confirmados mediante espectroscopía UV, proponiéndose tres probables flavonoides: dos de núcleo flavona y 1 de flavanona.

\section{REFERENCIAS BIBLIOGRÁFICAS}

1. Martínez S, González J, Culebras J, Tunón M. Flavonoides: propiedades y acciones antioxidantes. Nutr. Hosp. 2002; $17(6): 271-278$.

2. Fonnegra R, Jimenez S. Plantas medicinales aprobadas en Colombia. 2 ed. Medellín. Editorial Universidad de Antioquia.; 2007.p. 97-99.

3. Jaramillo J, Diaz C. El cultivo de las crucíferas Brócoli, Coliflor, Repollo, Col china en Colombia. Corporación Colombiana de Investigación Agropecuaria CORPOICA. Centro investigación La Selva; 2006 Septiembre. Documento de trabajo 2.1.4.04.32.05.

4. Lock O. Investigación fitoquímica. Métodos en el estudio de productos naturales. 2ed. Lima. Pontificia Universidad Católica del Perú Fondo Editorial. Lima; 1994. p. 98-102.

5. Stahl E. Thin-Layer Chromatography. A Laboratory Handbook. Berlin. Editorial Springer-Velarg; 1978. p. 687-703.

6. Mabry TJ, Markham KR, Thomas MB. The systematic identification of flavonoids. Springer-Verlag. New York-Heidelberg-Berlin, 1970.

7. Bonilla P, Arroyo J, Lozano N, Beltrán H, Alba A, Aguedo J, et al. Composición química y actividad farmacológica del extracto etanólico de Satureja sericea "goyal". Ciencia e Investigación 2011; 14(1): 14-20.

8. Czeczot, H. (2000). Biological activities of flavonoids: A review. Polish Journal of Food and Nutrition Sciences, 950,3-13.

9. Singh, J., Upadhyay, A.K., Prasad, K., Bahadur, A. and Rai, M. (2007). Variability of carotenes, vitamin C, E and phenolics in Brassica vegetables. Journal of Food Composition and Analysis, 20, 106-112.

10. Fernández Leon, M. Evolución de los parámetros de calidad físico-químico y funcional de distintas brassicas sometidas a diferentes tratamientos postcosecha. [Tesis doctoral]. España: Repositorio institucional Universidad de Extremadura;2013.

\section{ANEXO}

Ficha taxonómica de la planta "col morada" 
Fernández, G. et al. Estudio etnobotánico y fitoquímico de hojas de Brassica oleracea L. "col morada".

UNIVERSIDAD NACIONAL MAYOR DE SAN MARCOS

Universidad del Perú, DECANA DE AMÉ RICA MUSEO DE HISTORIA NATURAL

"Año de la Promoción de la Industria Responsable y del Compromiso Climático"

CONSTANCIA No 256- USM-2014

LA JEFA DEL HERBARIO SAN MARCOS (USM) DEL MUSEO DE HISTORIA NATURAL, DE LA UNIVERSIDAD NACIONAL MAYOR DE SAN MARCOS, DEJA CONSTANCIA QUE:

La muestra vegetai (planta completa) recibida de Gustavo FERNANDEZ REBAZA, Jorge TOLENTINO CHAVEZ, María QUIÑONES HUAYANEY, Jose AYME

HUAMANí, Yoselin CHAMBI VELASQUEZ y Pablo BONILLA RIVERA, ha sido estudiada y clasificada como: Brassica oleracea $L$. y tiene la siguiente posición taxonómica, segúr. el Sistema de Clasificación de Cronquist (1988).

\section{DIVISION: MAGNOLIOPHYTA}

\section{CLASE: MAGNOLIOPSIDA}

SUBCLASE: DILLENIIDAE

ORDEN: CAPPARALES

FAMILIA: BRASSICACEAE

\section{GENERO: Brassica}

ESPECIE: Brassica oleracea $L$.

Nombre vulgar: "col morada"

Determinado por Mag. Hamilton Beltran.

Se extiende la presente constancia a solicitud de la parte interesada, para fines de estudios.

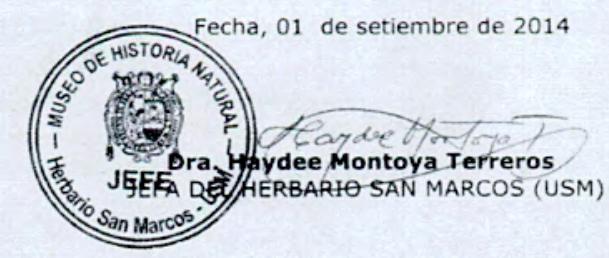

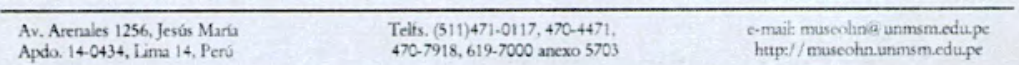

